\title{
On Lorentezian almost Para-contact Manifold
}

\author{
Vinod Chandra $^{1} \cdot$ Shankar Lal ${ }^{2}$ \\ ${ }^{1}$ Department of Mathematics, Uttaranchal University, Dehradun (UK), India \\ ${ }^{2}$ HNBGU, S. R. T. Campus, Badshahithaul, Tehri Garhwal, Uttarakhand, INDIA, \\ *Corresponding Author E-mail: chandravinod8126@gmail.com
}

Received: 26.5.2021; Revised:24.8.2021; Accepted: 15.10.2021

CSociety for Himalayan Action Research and Development

\begin{abstract}
In the present paper, we focus on Lorentzian almost para-contact manifold and explain their relationship. In section 1, we have introduced the historical background of a contact manifold. Next, in section 2 , we have studied the basic formulae of the Lorentzian metric manifold. Further, in section 3, we introduced a new tensor field $h$ as well as calculated some theorems and lemma. Now in section 4, we have investigated curvature properties and their relationship with the Lorentzian almost para-contact manifold. In the end section, we have discussed the entire work.
\end{abstract}

AMS Mathematics subject classification (2010): 53D12, 53C15, 53C25

Key Words: Contact structure, Almost contact structure, Lorentzian metric

\section{Introduction}

In differential geometry, contact structure manifold is a well known and highly studied research field. The notion of contact geometry of manifold has evolved from the mathematical formalism of classical mechanics. The contact structure was studied first by Takashi (1969) with compatible pseudo-Riemannian metrics. The almost para contact structure on pseudo-
Riemannian manifold $\mathrm{M}$ of dimension $(2 \mathrm{n}+1)$ is defined in (1985) and the almost para complex structure on $M(2 n+1) \times R$ is constructed. Duggal (1990) studied contact Lorentzian structure for physical relevance in spacetime. The systematic study of Lorentzian contact manifold was undertaken by the present author G. Calvaruso (2007 a, 2007 b). The survey of Lorentzian almost para-contact manifold was initiated by K. Matsumoto (1989).

\section{Basic Formulae}

Let us take $M$ to be a $(2 n+1)$ dimensional Lorentzian metric manifold. Then a structure $(\varphi, \xi, \eta)$ constituted by a $(1,1)$ tensor $\varphi$, vector field $\xi$ and a 1 -form $\eta$, such that

$$
\begin{aligned}
& \varphi^{2}=I d+\eta \otimes \xi \\
& \eta(\xi)=-1, \\
& \eta \circ \varphi=0 \quad, \varphi(\xi)=0 .
\end{aligned}
$$

Let $\mathrm{g}$ is a Lorentzian metric $M^{2 n+1}$, and then $\mathrm{g}$ is compatible with $M^{2 n+1}$ Lorentzian metric manifold $(\varphi, \xi, \eta)$ if 


$$
g(\varphi \mathrm{X}, \varphi \Upsilon)=g(\mathrm{X}, \Upsilon)+\eta(\mathrm{X}) \eta(\Upsilon)
$$

Then the structure $(\varphi, \xi, \eta)$ is compatible with a metric $g$ called a Lorentzian almost para-contact manifold. Consider that from equation (2.1) to (2.4) we have $\eta(\mathrm{X})=g(\mathrm{X}, \xi)$ and $\Phi(\mathrm{X}, \Upsilon)=g(\mathrm{X}, \varphi \Upsilon)=g(\varphi \mathrm{X}, \Upsilon)=\Phi(\Upsilon, \mathrm{X})$ in particular $g(\xi, \xi)=-1$ and equation (2.4) implies that $g(\varphi \mathrm{X}, \Upsilon)=g(\mathrm{X}, \varphi \Upsilon)$.

Definition 1.1: A Lorentzian almost para-contact manifold $M^{2 n+1}$ will be Lorentzian para-Kenmotsu manifold if:

$$
\begin{aligned}
& \left(\nabla_{\mathrm{X}} \varphi\right) \Upsilon=-g(\varphi \mathrm{X}, \Upsilon) \xi-\eta(\Upsilon) \varphi \mathrm{X} \\
& \nabla_{\mathrm{X}} \xi=-\mathrm{X}-\eta(\mathrm{X}) \xi \\
& \left(\nabla_{\mathrm{X}} \eta\right) \Upsilon=-g(\mathrm{X}, \Upsilon)-\eta(\mathrm{X}) \eta(\Upsilon) .
\end{aligned}
$$

Let us consider $M^{2 n+1} \times R$ Manifold. Let us denote $\left(\mathrm{X}, f \frac{d}{d t}\right)$ a vector field, $f$ is a $C^{\infty}$ function and $\mathrm{X}$ is tangent to the manifold. An almost complex structure is defined by

$$
J\left(X, f \frac{d}{d t}\right)=\left(\varphi \mathrm{X}-f \xi, \eta(\mathrm{X}) \frac{d}{d t}\right),
$$

here necessary and sufficient condition for integrability is that Nijenhuis torsion of $\mathbf{J}$ must vanishes

$$
\begin{array}{r}
{[J, J]((\mathrm{X}, 0),(\Upsilon, 0))=-([\mathrm{X}, \Upsilon], 0)+\left[\left(\varphi \mathrm{X}, \eta(\mathrm{X}) \frac{d}{d t}\right),\left(\varphi \Upsilon, \eta(\Upsilon) \frac{d}{d t}\right)\right]} \\
-J\left[\left(\varphi \mathrm{X}, \eta(\mathrm{X}) \frac{d}{d t}\right),(\Upsilon, 0)\right]-J\left[(\mathrm{X}, 0),\left(\varphi \Upsilon, \eta(\Upsilon) \frac{d}{d t}\right)\right] \\
{[J, J]\left((\mathrm{X}, 0),\left(0, \frac{d}{d t}\right)\right)=\left[\left(\varphi \mathrm{X}, \eta(\mathrm{X}) \frac{d}{d t}\right),(-\xi, 0)\right]} \\
-J\left[\left(\varphi \mathrm{X}, \eta(\mathrm{X}) \frac{d}{d t}\right),\left(0, \frac{d}{d t}\right)\right]-J[(\mathrm{X}, 0),(-\xi, 0)] .
\end{array}
$$

Now we define four Nijenhuis tensor $N^{1}, N^{2}, N^{3}$ and $N^{4}$ as
(a) $N^{1}=[\varphi, \varphi]+2 d \eta \otimes \xi$,
(b) $N^{2}(\mathrm{X}, \Upsilon)=\left(L_{\varphi \mathrm{X}} \eta\right) \Upsilon-\left(L_{\varphi \Upsilon} \eta\right) \mathrm{X}$, 

(c) $N^{3}=\left(L_{\xi} \varphi\right) \mathrm{X}$,
(d) $N^{4}=\left(L_{\xi} \eta\right) \mathrm{X}$.

So we have seen that the almost contact structure is normal if these four tensors vanishes.

Lemma 1.1: Let us take almost contact structure $(\varphi, \xi, \eta)$ with pseudo-Riemannian metric g, we have

$$
\begin{array}{rl}
2 g\left(\left(\nabla_{\mathrm{X}} \varphi\right) \Upsilon, \mathrm{Z}\right)=3 d & d(\mathrm{X}, \varphi \Upsilon, \varphi \mathrm{Z})-3 d \Phi(\mathrm{X}, \Upsilon, \mathrm{Z})+g\left(N^{1}(\Upsilon, \mathrm{Z}), \varphi \mathrm{X}\right) \\
& +N^{2}(\Upsilon, \mathrm{Z}) \eta(\mathrm{X})+2 d \eta(\mathrm{X}, \varphi \Upsilon) \eta(\mathrm{Z})+2 d \eta(\mathrm{X}, \varphi \mathrm{Z}) \eta(\Upsilon)
\end{array}
$$

Proof: Levi-Civita connection $\nabla$ for $g$ is given by

$$
\begin{aligned}
2 g\left(\left(\nabla_{\mathrm{X}} \varphi\right) \Upsilon, \mathrm{Z}\right)=\mathrm{X} g(\Upsilon, \mathrm{Z}) & +\Upsilon g(\mathrm{X}, \mathrm{Z})-\mathrm{Zg}(\mathrm{X}, \Upsilon)+g([\mathrm{X}, \Upsilon], \mathrm{Z}) \\
- & g([\Upsilon, \mathrm{Z}], \mathrm{X})+g([\mathrm{Z}, \mathrm{X}], \Upsilon) .
\end{aligned}
$$

Therefore we have

$$
\begin{aligned}
2 g\left(\left(\nabla_{\mathrm{X}} \varphi\right) \Upsilon, \mathrm{Z}\right) & =2 g\left(\nabla_{\mathrm{X}} \varphi \Upsilon, \mathrm{Z}\right)+2 g\left(\nabla_{\mathrm{X}} \Upsilon, \varphi \mathrm{Z}\right) \\
= & \mathrm{X} g(\varphi \Upsilon, \mathrm{Z})+\varphi \Upsilon g(\mathrm{X}, \mathrm{Z})-\mathrm{Z} g(\mathrm{X}, \varphi \Upsilon)+g([\mathrm{X}, \varphi \Upsilon], \mathrm{Z}) \\
& +g([\mathrm{Z}, \mathrm{X}], \varphi \Upsilon)-g([\varphi \Upsilon, \mathrm{Z}], \mathrm{X})+\mathrm{X} g(\Upsilon, \varphi \mathrm{Z})+\Upsilon g(\mathrm{X}, \varphi \mathrm{Z}) \\
& -\varphi \mathrm{Z} g(\mathrm{X}, \Upsilon)+g([\mathrm{X}, \Upsilon], \varphi \mathrm{Z})+g([\varphi \mathrm{Z}, \mathrm{X}], \Upsilon)-g([\Upsilon, \varphi \mathrm{Z}], \mathrm{X}) \\
= & \mathrm{X} \Phi(\Upsilon, \mathrm{Z})+\varphi \Upsilon \Phi(\varphi \mathrm{Z}, \mathrm{X})-\eta(\mathrm{X}) \eta(\mathrm{Z})-\mathrm{Z} \Phi(\mathrm{X}, \Upsilon)+\Phi([\mathrm{X}, \varphi \Upsilon], \varphi \mathrm{Z}) \\
- & \eta([\mathrm{X}, \varphi \Upsilon]) \eta(\mathrm{Z})+\Phi([\mathrm{Z}, \mathrm{X}], \Upsilon)-g(\varphi[\varphi \Upsilon, \mathrm{Z}], \varphi \mathrm{X})-\eta([\varphi \Upsilon, \mathrm{Z}]) \eta(\mathrm{X}) \\
+ & \mathrm{X} \Phi(\Upsilon, \mathrm{Z})+\Upsilon \Phi(\mathrm{X}, \mathrm{Z})-\varphi \mathrm{Z} \Phi(\varphi \Upsilon, \mathrm{X})+\eta(\mathrm{X}) \eta(\Upsilon)+\Phi([\mathrm{X}, \Upsilon], \mathrm{Z}) \\
+ & \Phi([\varphi \mathrm{Z}, \mathrm{X}], \varphi \Upsilon)-\eta([\varphi \mathrm{Z}, \mathrm{X}]) \eta(\Upsilon)+\{\Phi[\Upsilon, \mathrm{Z}], \mathrm{X}-g([\Upsilon, \mathrm{Z}], \varphi \mathrm{X})\} \\
- & \{\Phi([\varphi \Upsilon, \varphi \mathrm{Z}], \mathrm{X})+\eta([\Upsilon, \varphi \mathrm{Z}]) \eta(\mathrm{X})-g(\varphi[\Upsilon, \varphi \mathrm{Z}], \varphi \mathrm{X}) \\
& -g([\varphi \Upsilon, \varphi \mathrm{Z}], \varphi \mathrm{X})\}+\{g(2 d \eta(\Upsilon, \mathrm{Z}) \xi, \varphi \mathrm{X})\} \\
= & 3 d \Phi(\mathrm{X}, \varphi \Upsilon, \varphi \mathrm{Z})-3 d \Phi(\mathrm{X}, \Upsilon, \mathrm{Z})+g\left(N^{1}(\Upsilon, \mathrm{Z}), \varphi \mathrm{X}\right) \\
& +N^{2}(\Upsilon, \mathrm{Z}) \eta(\mathrm{X})+2 d \eta(\mathrm{X}, \varphi \Upsilon) \eta(\mathrm{Z})+2 d \eta(\mathrm{X}, \varphi \mathrm{Z}) \eta(\Upsilon) \\
&
\end{aligned}
$$


If the Lorentzian metric $\mathrm{g}$ (compatible) satisfies

$$
g(\mathrm{X}, \varphi \Upsilon)=g(\varphi \mathrm{X}, \Upsilon)=d \eta(\mathrm{X}, \Upsilon)
$$

Then the structure $(M, \varphi, \xi, \eta)$ is called Lorentzian almost para-contact manifold.

From equation (2.1), (2.2) and (2.7) we have $d \eta(\mathrm{X}, \xi)=g(\mathrm{X}, \varphi \xi)=g(\varphi \xi, \mathrm{X})=0$ and now denoting $\mathrm{L}$ the Lie derivative,

$$
N^{4}=L_{\xi} \eta=d \eta(\xi)+d \eta(., \xi)=0
$$

since we know, $\eta(\mathrm{X})=g(\mathrm{X}, \xi)$, from $L_{\xi} \eta=0$ we get

$$
\left(L_{\xi} \eta\right)=0=\xi g(\mathrm{X}, \xi)-g([\xi, \mathrm{X}], \xi)=g\left(\mathrm{X}, \nabla_{\xi} \xi\right)
$$

for any vector $\mathrm{X}$. and also $\nabla_{\xi} \xi=0$, using (2.13) we get

$$
\begin{aligned}
\left(L_{\varphi \mathrm{X}} \eta\right) \Upsilon & =(\varphi \mathrm{X})(\eta(\Upsilon))-\Upsilon \eta(\varphi \mathrm{X})-\eta[\varphi \mathrm{X}, \Upsilon] \\
& =2 d \eta(\mathrm{X}, \varphi \Upsilon)=2 g(\varphi \mathrm{X}, \varphi \Upsilon)
\end{aligned}
$$

So $N^{2}(\mathrm{X}, \Upsilon)=2 d \eta(\varphi \mathrm{X}, \Upsilon)-2 d \eta(\mathrm{X}, \varphi \Upsilon)=0$, at once $d \Phi=0$. Therefore Lemma (1.1) implies the following:

Theorem 2.1: In the Lorentzian almost para-contact manifold $(M, \varphi, \xi, \eta, g)$ we have

$$
2 g\left(\left(\nabla_{\mathrm{X}} \varphi\right) \Upsilon, \mathrm{Z}\right)=g\left(N^{1}(\Upsilon, \mathrm{Z}), \varphi \mathrm{X}\right)+2 d \eta(\mathrm{X}, \varphi \Upsilon) \eta(\mathrm{Z})+2 d \eta(\mathrm{X}, \varphi \mathrm{Z}) \eta(\Upsilon)
$$

\section{The Tensor Field $h$}

Let's introduce a tensor

$$
h=\frac{1}{2} L_{\xi} \varphi=\frac{1}{2} N^{3}
$$

Lemma 3.1: On a contact manifold following results holds

$$
\begin{aligned}
& \nabla_{\xi} \varphi=0, \\
& \nabla_{\mathrm{X}} \xi=-\varphi \mathrm{X}-\varphi h \mathrm{X},
\end{aligned}
$$

where $h$ is symmetric operator, if $h$ anti-commutes with $\varphi$ then $\operatorname{tr} h=0$. 
Theorem 3.1: In a Lorentzian almost-para contact manifold, $h$ is symmetric operator, then

$$
\begin{aligned}
& \nabla_{\xi} \varphi=0, \\
& \nabla_{\mathrm{X}} \xi=\varphi \mathrm{X}+\varphi h \mathrm{X},
\end{aligned}
$$

Proof: On using Lemma. 1.1, we have

$$
\begin{aligned}
2 g\left(\left(\nabla_{\mathrm{X}} \varphi\right) \xi, \mathrm{Z}\right) & =g\left(\varphi^{2}[\xi, \mathrm{Z}]-\varphi[\xi, \varphi \mathrm{Z}], \varphi \mathrm{X}\right)-2 d \eta(\varphi \mathrm{Z}, \mathrm{X}) \\
= & -g\left(\varphi\left(L_{\xi} \varphi\right) \mathrm{Z}, \varphi \mathrm{X}\right)-2 g(\varphi \mathrm{Z}, \varphi \mathrm{X}) \\
= & -g\left(\left(L_{\xi} \varphi\right) \mathrm{Z}, \mathrm{X}\right)+\eta\left(\left(L_{\xi} \varphi\right) \mathrm{Z}\right) \eta(\mathrm{X})-2 g(\mathrm{Z}, \mathrm{X})+2 \eta(\mathrm{Z}) \eta(\mathrm{X}) \\
= & -g\left(\left(L_{\xi} \varphi\right) \mathrm{X}, \mathrm{Z}\right)-2 g(\mathrm{X}, \mathrm{Z})+2 g(\eta(\mathrm{X}) \xi, \mathrm{Z})
\end{aligned}
$$

and hence $-\varphi \nabla_{\mathrm{X}} \xi=-\frac{1}{2}\left(L_{\xi} \varphi\right) \mathrm{X}-\mathrm{X}+\eta(\mathrm{X}) \xi$ applying $\varphi$ we get

$$
\nabla_{\mathrm{X}} \xi=\varphi \mathrm{X}+\varphi h \mathrm{X}
$$

for anti-commutative, note that

$$
\begin{gathered}
2 g(\mathrm{X}, \varphi \Upsilon)=2 g(\varphi \mathrm{X}, \Upsilon)=2 d \eta(\mathrm{X}, \Upsilon)=g\left(\Upsilon, \nabla_{\mathrm{X}} \xi\right)-g\left(\mathrm{X}, \nabla_{\Upsilon} \xi\right) \\
=g(\Upsilon, \varphi \mathrm{X}+\varphi h \mathrm{X})-g(\mathrm{X}, \varphi \Upsilon+\varphi h \Upsilon)
\end{gathered}
$$

from this, we have $g(\Upsilon, \varphi h \mathrm{X})-g(h \varphi \mathrm{X}, \Upsilon)=0$ which gives $h \varphi-\varphi h=0$ and hence $h=0$.

Definition 3.1: A contact Lorentzian manifold $(M, \eta, g)$ is assumed to be

(i) Sasakian Manifold if it is normal.

(ii) $\mathrm{K}$-contact Manifold, if $\xi$ is a killing vector.

Theorem 3.2: An Lorentzian almost para-contact manifold $M^{2 n+1}$ will be Lorentzian para Sasakian manifold if:

$$
\left(\nabla_{\mathrm{X}} \varphi\right) \Upsilon=g(\mathrm{X}, \Upsilon) \xi+\eta(\Upsilon) \mathrm{X}+2 \eta(\mathrm{X}) \eta(\Upsilon) \xi
$$

If we take $\Upsilon=\xi$ in the above equation, we have the theorem.

Corollary 3.1: If the tensor field $h=0$ then a Lorentzian para Sasakian manifold is K-(para) contact. 


\section{Curvature Properties of Lorentezian Contact Manifold}

Now we investigate curvature properties of a Lorentzian almost para-contact Manifold $(M, \eta, g)$. Let us denote $\mathrm{R}$ by its curvature tensor of $M^{2 n+1}$, using curvature tensor

$$
R(\xi, \mathrm{X}) \xi=\nabla_{\xi} \nabla_{\mathrm{X}} \xi-\nabla_{\mathrm{X}} \nabla_{\xi} \xi-\nabla_{[\xi, \mathrm{X}]} \xi
$$

Using the condition of Lorentzian almost para-contact, $\nabla_{\mathrm{X}} \xi=\varphi \mathrm{X}+\varphi h \mathrm{X}$,

$$
R(\xi, \mathrm{X}) \xi=\nabla_{\xi}(\varphi \mathrm{X}+\varphi h \mathrm{X})+\varphi[\xi, \mathrm{X}]+\varphi h[\xi, \mathrm{X}]
$$

Applying $\varphi$ and recalling that $\nabla_{\xi} \xi=0$ we have

$$
\begin{aligned}
\varphi(R(\xi, \mathrm{X}) \xi) & =\nabla_{\xi}(\mathrm{X}+h \mathrm{X})+\eta\left(\nabla_{\xi}(\mathrm{X}+h \mathrm{X})\right) \xi+[\xi, \mathrm{X}]+\eta([\xi, \mathrm{X}]) \xi-h[\xi, \mathrm{X}] \\
= & \left(\nabla_{\xi} h\right) \mathrm{X}-\nabla_{\mathrm{X}} \xi-h \nabla_{\mathrm{X}} \xi,
\end{aligned}
$$

Using (3.5) and $\varphi h-h \varphi=0$ this becomes

$$
\varphi(R(\xi, \mathrm{X}) \xi)=\left(\nabla_{\xi} h\right) \mathrm{X}-\varphi \mathrm{X}+2 h \varphi \mathrm{X}-h^{2} \varphi \mathrm{X}
$$

This equation gives the theorem as,

Theorem 4.1: on a Lorentzian almost para-contact manifold we get the following formulae

$$
\left(\nabla_{\xi} h\right) \mathrm{X}=\varphi \mathrm{X}+2 \varphi h \mathrm{X}+h^{2} \varphi \mathrm{X}-\varphi(R(\mathrm{X}, \xi) \xi)
$$

Corollary 4.1: A Lorentzian almost para-contact manifold is K-contact if the sectional curvature of all sectional plane containing $\xi=1$.

Now in a K-contact manifold,

$$
R_{\mathrm{X} \xi} \xi=\mathrm{X}+\eta(\mathrm{X}) \xi
$$

Proof: If the structure is K-contact it's well known that then [since $\nabla_{X} \xi=\varphi \mathrm{X}$ ] we have for $\mathrm{X}$ orthogonal to $\xi$

$$
R_{\xi \mathrm{X}} \xi=\nabla_{\xi}(\varphi \mathrm{X})-\varphi[\xi, \mathrm{X}]=\varphi \nabla_{\mathrm{X}} \xi=\varphi^{2} \mathrm{X}=\mathrm{X}
$$

from this expression proof is evident. 
Theorem 4.2: A Lorentzian almost para-contact manifold $M^{2 n+1}$ is K contact if it satisfies the curvature condition $Q(\xi, \xi)=2 n$.

Proof: We start with curvature tensor R of the Manifold $M^{2 n+1}$, taken sign convention

$$
R(X, Y)=\nabla_{\mathrm{X}} \nabla_{\Upsilon}-\nabla_{[\mathrm{X}, \Upsilon]} \cdot
$$

Using (3.5) and $\nabla_{\xi} \varphi=0$, we find

$$
R(\mathrm{X}, \xi) \xi=\nabla_{\xi}(\varphi \mathrm{X}+\varphi h \mathrm{X})-\varphi[\mathrm{X}, \xi]+\varphi h[\mathrm{X}, \xi]=\varphi\left(\left(\nabla_{\xi} h\right)(\mathrm{X})\right)-\varphi^{2} \mathrm{X}+h^{2} \mathrm{X}
$$

That is

$$
\ell:=R(\cdot, \xi) \xi=\varphi\left(\nabla_{\xi} h\right)-\varphi^{2}+h^{2}
$$

Applying $\varphi$ in the above equation, we get

$$
\varphi \ell \mathrm{X}=\left(\nabla_{\xi} h\right) \mathrm{X}-\varphi \mathrm{X}-h^{2} \varphi \mathrm{X}
$$

Which implies that

$$
\varphi \ell \varphi \mathrm{X}=\left(\left(\nabla_{\xi} h\right) \varphi\right) \mathrm{X}-\varphi^{2} \mathrm{X}-h^{2} \mathrm{X}
$$

So we get

(4.13) $\varphi \ell \varphi-\ell=2\left(\varphi^{2}\right)$.

The sectional curvature is given by

$$
\begin{aligned}
& K\left(\xi, e_{i}\right)=-R\left(\xi, e_{i}, \xi, e_{i}\right)=g\left(\ell e_{i}, e_{i}\right), \text { and } \\
& K\left(\xi, \varphi e_{i}\right)=-R\left(\xi, \varphi e_{i}, \xi, \varphi e_{i}\right)=-g\left(\varphi \ell \varphi e_{i}, e_{i}\right)
\end{aligned}
$$

Take $\left\{\xi, e_{1}, \ldots \ldots \ldots . e_{n}, \varphi e_{1}, \ldots \ldots \ldots . ., \varphi e_{n}\right\} \varphi$-the basis of vector fields on $M^{2 n+1}$, then by (4.13)

$$
Q(\xi, \xi)=-\sum_{i=1}^{n}\left(K\left(\xi, e_{i}\right)+K\left(\xi, \varphi e_{i}\right)\right)=2 n-\operatorname{tr} h^{2}
$$

We know that the condition characterizes K-contact manifold $Q(\xi, \xi)=2 n$, it implies that $\operatorname{tr} h^{2}=0$ and so $h=0$. So we have the theorem. 


\section{Conclusion}

In this article, we have concluded some results of Lorentzian almost para-contact manifold and their properties. A Lorentzian almost paracontact manifold is $\mathrm{K}$ contact iff satisfies the condition $Q(\xi, \xi)=2 n$. And some other consequences for sectional curvature and tensor field $h$. Some definitions and theorems also discussed related to the more complicated structure of manifolds.

Acknowledgement: All authors are grateful to the referee for his valuable suggestion for the improvement of the research paper.

\section{References}

Calvaruso G (2007a). Homogeneous structures on three-dimensional Lorentzian manifolds, J. Geom. Phys. Vol. 57, pp. 1279-1291

Calvaruso G (2007b). Einstein-like metrics on three-dimensional homogeneous Lorentzian manifolds, Geom. Dedicata vol. 127, pp. 99-119.

Duggal K L (1990). Spacetime manifolds and contact structures, Int. J. Math. Math. Sci. vol. 13, pp. 545-554.

Kaneyuki S, Willams F. L (1985). Almost paracontact and parahodge structures on manifolds, Nagoya Math. J. 99173-187.

Matsumoto K (1989). Bull. Yamagata Univ., Nat. Sci. vol. 12, pp. 151-156.

Takahashi T (1969). Sasakian manifold with pseudo-Riemannian metrics, Tôhoku Math. J. vol. 21, pp.271-290. 